\title{
UV Radiation Exposure
}

National Cancer Institute

\section{Source}

National Cancer Institute. UV Radiation Exposure. NCI Thesaurus. Code C44462.

The exposure to uv radiation via incident light from the sun. 